\title{
Superadditivity and Subadditivity in Fair Division
}

\author{
Rishi S. Mirchandani ${ }^{1}$ \\ ${ }^{1}$ Fox Chapel Area Senior High School, Pittsburgh, PA, USA \\ Correspondence: Rishi Mirchandani, Fox Chapel Area Senior High School, Pittsburgh, PA 15238, USA. E-mail: \\ mirchandani.rs@gmail.com
}

Received: July 12, 2013 Accepted: August 5, 2013 Online Published: August 13, 2013

doi:10.5539/jmr.v5n3p78 ＵRL: http://dx.doi.org/10.5539/jmr.v5n3p78

\begin{abstract}
I examine the classic problem of fair division of a piecewise homogeneous good. Previous work developed algorithms satisfying various combinations of fairness notions (such as proportionality, envy-freeness, equitability, and Pareto-optimality). However, this previous work assumed that all utility functions are additive. Recognizing that additive functions accurately model utility only in certain situations, I investigate superadditive and subadditive utility functions. Next, I propose a new division protocol that utilizes nonlinear programming and test it on a sample instance. Finally, I prove theoretical results that address (a) relationships between fairness notions, and (b) the orthogonal issue of division efficiency (i.e., the price of satisfying particular fairness notions).
\end{abstract}

Keywords: fair division, fairness notions, utility functions, superadditivity and subadditivity, mathematical programming, price of fairness

\section{Introduction}

The problem of fair division of goods dates back to ancient times (Note 1) and occurs frequently in the social sciences, law, economics, game theory, and other fields. The work of Steinhaus, Knaster and Banach in the 1940s formalized the problem using the famous "cake-cutting" abstraction: there are $m \geq 2$ players who must distribute a single piecewise homogeneous "cake" amongst themselves in a fair manner. (Assuming that the cake is piecewise homogeneous means that there are $n \geq 1$ distinct components, each of which is uniform within itself.) The different players may have different utilities, or levels of value, for fractional allotments of the various components. For example, one player may prefer a third of the strawberry portion to half of the chocolate portion, and a second player may prefer a fourth of the chocolate portion to the entire strawberry portion, while a third player might derive positive utility from only the rhubarb portion. (Such a set of player-dependent utility functions for each homogenous piece defines an instance of the cake-cutting problem.) The objective is to obtain a fair division of the cake. There are two immediate difficulties.

Defining fairness. The first difficulty is that there are several possible definitions for what constitutes a "fair" division. The notions of proportionality, envy-freeness, equitability, and Pareto-optimality (which are formally defined in Section 1.2) offer plausible answers; however, it is only guaranteed that all four notions can concurrently be satisfied when there are two players and their utility functions are additive (see Brams, 2008). Thus, the arbitrators may be forced to make a choice between these different fairness notions. Steinhaus (1948), Brams and Taylor (1995), and Peterson and Su (2002) are a few among many who have found division protocols, which yield allocations satisfying various combinations of the fairness notions for instances with all additive utility functions.

Efficiency. While fairness is clearly a major consideration in the division of goods, it is not the only one. Recent research has highlighted the importance of the aggregate social welfare resulting from the allocation. A second difficulty in the cake-cutting problem thus arises: while an allocation may be, e.g., envy-free, it may also be inefficient; in other words, the total social welfare derived from the allocation may be lower than the maximum possible social welfare for the given instance. Caragiannis et al. (2012) and Aumann and Dombb (2011) use the concept of price of fairness to quantify the decrease in efficiency when the optimal allocation, which maximizes total societal utility, is modified to meet a fairness criterion. (I provide a formal definition of the price of fairness in Section 1.2.) 


\subsection{Problem Statement and Overview of Results}

A critical assumption underlying many previous results is that utility functions are universally additive. However, there are many situations where additive functions do not accurately model utility. For example, one would not necessarily value two slices of chocolate cake twice as much as one slice. Allowing for utility functions to be superadditive or subadditive introduces many more wrinkles into the problem. I show that, when utilities are nonadditive, some division protocols developed in previous works fail to meet the fairness notions for which they were designed. As a solution, I propose an alternative mathematical programming protocol in which fairness notions are defined by nonlinear constraints. Additionally, I prove that with superadditive and subadditive utility functions, the price of fairness can be unbounded. In a corollary to this result, I show that proportionality, which for instances with all additive functions is easily met and even trivial, may be impossible to achieve.

I demonstrate that my modeling framework can also easily incorporate interactions between the components, another concept that the related literature appears to have neglected. Finally, I implement my model on commercial mathematical programming software and present sample results to demonstrate the effectiveness of my method.

\subsection{Definitions, Terminology, and Notations}

Recall that the cake must be divided amongst $m$ players and consists of $n$ components. I denote by $M$ the set $\{1,2, \ldots, m\}$ and by $N$ the set $\{1,2, \ldots, n\}$.

Utility Functions. Each player $i$ has a set of utility functions $\left\{u_{i j}(\cdot)\right\}_{j=1}^{n}$. For all $i \in M$ and $j \in N, u_{i j}(\cdot)$ is defined on $[0,1]$ (Note 2$)$. An allocation $\mathbf{X}$ is defined by the matrix $\left[x_{i j}\right]_{m \times n}$, where $x_{i j}$ denotes the fraction of component $j$ that player $i$ receives. Note that for all $i \in M$ and $j \in N, 0 \leq x_{i j} \leq 1$, and that $x_{i j}$ is a measure of quantity of allotment (Note 3), not of utility. In contrast, $u_{i j}\left(x_{i j}\right)$ denotes the utility derived by $i$ from the fraction $x_{i j}$ of $j$ that she receives. I define $a_{i j}$ as the fraction of the whole cake (with respect to utility) that component $j$, in its entirety, represents to $i$. Accordingly, $a_{i j}=u_{i j}(1)$. Finally, as is customary in the literature, I assume that players' utility functions are normalized such that the entire cake gives each player a utility of 1 ; that is, $\sum_{j \in N} a_{i j}=1, \forall i \in M$.

Fairness Criteria. The leading mathematical definitions of fairness are proportionality, envy-freeness, equitability, and Pareto-optimality. An allocation $\mathbf{X}$ is proportional if and only if each player assigns her own allotment a value of at least $\frac{1}{m}$; formally, $\sum_{j \in N} u_{i j}\left(x_{i j}\right) \geq \frac{1}{m}, \forall i \in M$. $\mathbf{X}$ is envy-free if and only if no player has a higher utility for another player's allotment; formally, $\sum_{j \in N} u_{i j}\left(x_{i j}\right) \geq \sum_{j \in N} u_{i j}\left(x_{k j}\right), \forall i, k \in M ; i \neq k . \mathbf{X}$ is equitable if and only if all the players value each of their own allotments at the same fraction (Note 4); formally, $\sum_{j \in N} u_{i j}\left(x_{i j}\right)=$ $\sum_{j \in N} u_{k j}\left(x_{k j}\right), \forall i, k \in M . \mathbf{X}$ is Pareto-optimal if and only if there is no division that could increase the utility of a player without decreasing the utility of at least one other player; formally, there exists no Pareto-dominating allocation $\mathbf{Y} \neq \mathbf{X}$ such that $\sum_{i \in M} \sum_{j \in N} u_{i j}\left(y_{i j}\right)>\sum_{i \in M} \sum_{j \in N} u_{i j}\left(x_{i j}\right)$ and $\sum_{j \in N} u_{i j}\left(y_{i j}\right) \geq \sum_{j \in N} u_{i j}\left(x_{i j}\right), \forall i \in M$.

The Price of Fairness (Note 5). Let $\mathbf{Z}^{*}$ denote the most efficient allocation (i.e., the allocation that maximizes the sum of the utilities of all players) for an instance $I$. Let $\mathbf{C}^{*}$ denote the most efficient allocation for $I$ from the subset of allocations satisfying a fairness criterion $\psi \in$ \{proportionality, equitability, envy-freeness (Note 6). Then (following Caragiannis et al., 2012) the price of $\psi$ is defined by the ratio

$$
\frac{\sum_{i \in M} \sum_{j \in N} u_{i j}\left(z_{i j}^{*}\right)}{\sum_{i \in M} \sum_{j \in N} u_{i j}\left(c_{i j}^{*}\right)} .
$$

\subsection{Literature Review}

The fair division problem has roots from at least as far back as 500 CE (Note 7). The Talmud, for example, discusses several situations where multiple parties or individuals contest desirable goods, and it describes several different (though seemingly inconsistent) division protocols. Aumann and Maschler (1985) use an interesting game-theoretic approach to demonstrate the logic behind the division protocols employed in the Talmud.

The vast majority of previous work has considered desirable, divisible goods, and the underlying problem being addressed is appropriately called the "cake-division problem". In the commonplace "I cut, you choose" approach for $m=2$, one player cuts the cake into two shares, and the other person chooses one of the two shares. This approach is guaranteed to result in a proportional and envy-free allocation for instances with all additive utilities. Steinhaus (1948) was among the first to formally define and analyze the fair division problem. He was also the first researcher to generalize the "I cut, you choose" division protocol to more than two people (see Borgers, 2010). In a series of papers and books, Brams and co-authors (see, e.g., Brams, 2008) propose and analyze algorithms for the 
cake-division problem. Brams and Taylor (1995) develop the first known procedure for envy-free cake division for an arbitrary number of recipients. Another excellent book, Moulin (2003), provides a more mathematical treatment of the problem. Reijnierse and Potters (1998) introduce an element of asymmetry in envy-freeness that captures the notion of entitlement; this allows a player to derive a greater utility from the share of another, more "entitled" player than his own share but still be envy-free. They develop an iterative, network-flow-based approach that determines an allocation as close to optimal as desired. Borgers (2010) presents a fine summary of the concepts and algorithms relating to cake division problems.

The fair division problem has also been examined for situations where the contested object(s) are undesirable. Undesirable goods are also referred to as chores, and potential recipients prefer to minimize their allotment of the chores. Petersen and Su (2002) explore the problem of chore division, and develop an envy-free procedure that works for up to four individuals. Chore division can also be important from a policy perspective, and Traxler (2002) discusses how to distribute chores amongst nations to mitigate climate change due to global warming. While Traxler only qualitatively compares the different principles involved in the distribution of efforts and payments needed to tackle climate change, his work nonetheless highlights the importance of the fair division problem.

In the last couple of years, computer scientists, mathematicians, and operations researchers have begun to look at the welfare loss if a division protocol attempts to achieve fairness at an individual level rather than to maximize the total societal welfare. Caragiannis et al. (2012) define the prices of proportionality, envy-freeness, and equitability, and places bounds on this price. Aumann and Dombb (2011) extend this result to the case where distributions of the cake must be contiguous. Both these papers consider only additive utility functions-one of the results in the present paper demonstrates that the price is unbounded if utility functions are nonadditive.

\section{Utility Functions}

A utility function provides a quantitative model of the happiness of a particular player given the fraction she receives from a particular component.

\subsection{Additive Utility Functions}

As described in Section 1.2, define $a_{i j}$ as the fraction of the value of the whole cake that $i$ believes $j$ to be worth.

Assuming that all $u_{i j}$ are additive means that $u_{i j}(p)+u_{i j}(q)=u_{i j}(p+q)$ for all $i, j$ and for all $p, q$ with $0<p, q<1$ and $0<p+q \leq 1$. Accordingly, the $u_{i j}$ can be defined on [0,1] as follows: $u_{i j}(p)=p \cdot a_{i j}, \forall i \in M, \forall j \in N$.

The reader is invited to study Figure 1 . Note that the points $(0,0)$ and $\left(1, a_{i j}\right)$ are guaranteed for any utility function - additive or not. What is particular to additive utility functions is that all points between $(0,0)$ and $\left(1, a_{i j}\right)$ form a line.

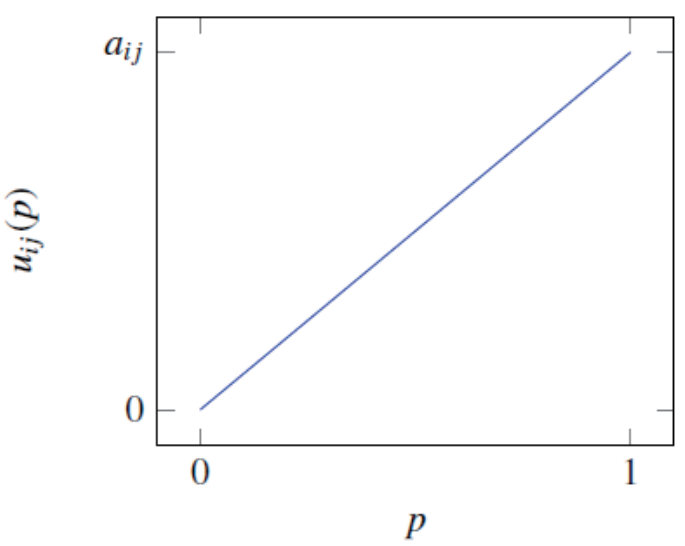

Figure 1. Additive utility function: $u_{i j}(p)=a_{i j} \cdot p$

I contend that it is uncommon, perhaps even rare, that an additive function is the most accurate model for utility. In the next section, I present a number of examples in which additive functions are unreasonable models of utility.

\subsection{Nonadditive Utility Functions}

Without the restriction of additivity, utility functions may be superadditive or subadditive (Note 8). 
Superadditivity. A superadditive utility function $\Lambda(\cdot)$ defined on $[0,1]$ has the property that $\Lambda(p+q) \geq \Lambda(p)+\Lambda(q)$ for all $p$ and $q$ with $0<p, q<1$ and $0<p+q \leq 1$. One case where such a function would be appropriate is when the object being divided is a medical resource, such as a single course of antibiotics. Taking half of the antibiotics yields negligible utility (Note 9) because the malicious bacteria are still prevalent enough for the disease to continue (Note 10). However, nearing the end of the course, utility increases dramatically as the bacteria population becomes increasingly smaller. Utility increases fastest as the allotment approaches 1 , at which point the bacteria population is eliminated.

Subadditivity. It is also possible to have the opposite scenario, where there is a saturation effect. This type of model is said to be subadditive (see Figure $2 \mathrm{~b}$ ). A subadditive utility function $\lambda(\cdot)$ defined on $[0,1]$ has the property that $\lambda(p+q) \leq \lambda(p)+\lambda(q)$ for all $p$ and $q$ with $0<p, q<1$ and $0<p+q \leq 1$. The cake example could be modeled by this type of function; utility increases rapidly with the first few bites. As the allotment approaches 1 , however, the rate of increase in utility becomes increasingly small as nourishment and satisfaction have already been attained.

Combination. A utility function may be superadditive on some subinterval(s) of the unit interval and subadditive on some other subinterval(s). For example, define a finite partition $\Pi=\left\{\pi_{1}, \ldots, \pi_{t}\right\}$ of cardinality $t$ of the unit interval. A twice-differentiable utility function $C(\cdot)$ exhibits both types of behavior if there exists $\Pi$ in which there is (1) at least one $r$ such that $C^{\prime \prime}(p)>0$ for $p \in \pi_{r}$ and (2) at least one $s$ such that $C^{\prime \prime}(p)<0$ for $p \in \pi_{s}$. As a practical example, consider a sigmoidal function as in Figure 2c. Such a function would be a good representation of the utility for say, time: a very small amount of time is of very little use; then, as the amount of time becomes more appropriate for a desired task to be completed, utility increases rapidly; finally, excess time adds negligible additional utility.

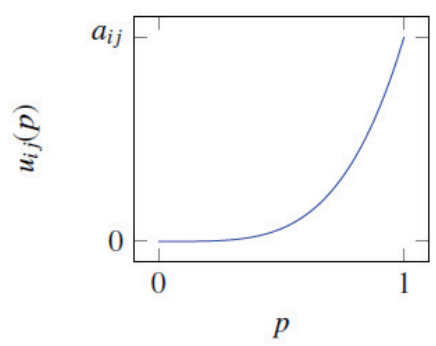

(a) A Superadditive Utility Function: $u_{i j}(p)=a_{i j} \cdot p^{4}$

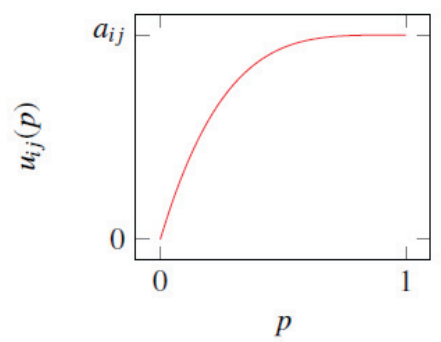

(b) A Subadditive Utility Function: $u_{i j}(p)=a_{i j} \cdot\left[1-(1-p)^{4}\right]$

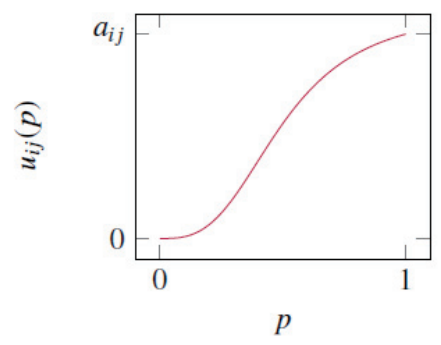

(c) A Sigmoidal Utility Function: $u_{i j}(p)=a_{i j} \cdot \frac{9 p^{3}}{8 p^{3}+1}$

Figure 2. Examples of various types of nonadditive utility functions

\subsection{Other Possibilities for Utility Functions}

Nonadditivity in utility functions introduces the possibility that the functions are not strictly monotonic on the entire domain; that is, it is not necessary that $u_{i j}^{\prime}(p)>0 \forall 0<p<1$ (Note 11).

It immediately follows that constraints on the range of $u_{i j}$ are also not necessary; it is not necessary that $0 \leq$ $u_{i j}(p) \leq 1 \forall 0<p<1$. The utility functions could even be discontinuous. Figure 3 depicts such utility functions. Following are some illustrative examples.

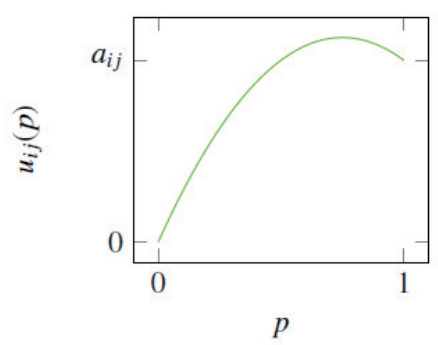

(a) Utility exceeds $a_{i j}$

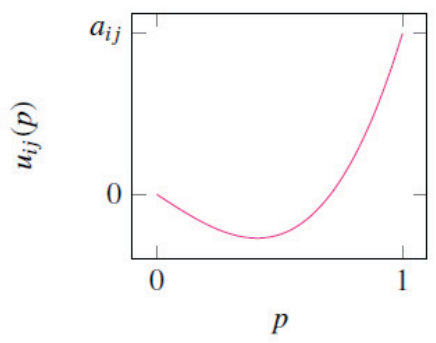

(b) Utility falls below 0

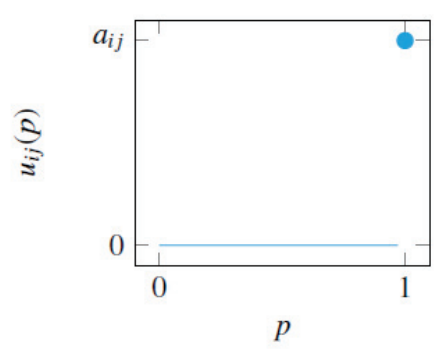

(c) Discontinuous Utility

Figure 3. Examples of utility functions with more eccentric behavior 
- The utility function in Figure 3a could apply to a medicine: once the allotment becomes an overdose, utility declines.

- The utility function in Figure $3 \mathrm{~b}$ when an heir is left with a tract of farmland that is too small to be economically irrigated but which still incurs taxes. As the land size increases, economies of scale make it increasingly profitable to farm the land.

- The discontinuous utility function in Figure 3c might apply when a valuable item is considered indivisible by a certain player. For example, Borgers (2010) discusses division of a collection of Agatha Christie novels. To some players, a few books out of the collection may be sufficient to yield a measurably large amount of utility. For others, however, an allotment of the books may yield no utility unless it is the entire collection (Note 12).

The constraints that I do maintain in this work are that, for all $i \in M$ and $j \in N$,

1) $0 \leq x_{i j} \leq 1$

2) $u_{i j}(1)=a_{i j}$, and

3) $\sum_{j \in N} a_{i j}=1$.

\section{Main Theoretical Results}

In previous literature where it is assumed that utilities are additive, the proportionality criterion has often been regarded as weak on the basis that any envy-free division is proportional. The logic in these works (e.g., Brams, 2010) is that a player who has a utility less than $\frac{1}{m}$ for her allotment must consider the remainder of the cake to have an aggregate utility greater than $\frac{m-1}{m}$, and accordingly she must consider at least one other player's allotment to be worth more than her own. The following theorem shows that, with nonadditive utility functions, this property does not necessarily hold.

Theorem 1 With nonadditive $u_{i j}$, an envy-free division is not necessarily proportional even if the whole cake is distributed. In fact, a proportional division may not even exist.

Proof. Consider an instance with $u_{i j}$ strictly convex for all $i \in M$ and $j \in N$. For an envy-free allocation, set all $x_{i j}=\frac{1}{m}$. Then the utility of any player $i$ is

$$
\sum_{j=1}^{n} u_{i j}\left(x_{i j}\right)=\sum_{j=1}^{n} u_{i j}\left(\frac{1}{m}\right)=\sum_{j=1}^{n} u_{i j}\left[\left(1-\frac{1}{m}\right)(0)+\left(\frac{1}{m}\right)(1)\right]
$$

By the definition of strict convexity,

$$
\sum_{j=1}^{n} u_{i j}\left[\left(1-\frac{1}{m}\right)(0)+\left(\frac{1}{m}\right)(1)\right]<\sum_{j=1}^{n}\left[\left(1-\frac{1}{m}\right)\left(u_{i j}(0)\right)+\left(\frac{1}{m}\right)\left(u_{i j}(1)\right)\right] .
$$

The latter expression may be manipulated as follows:

$$
\sum_{j=1}^{n}\left[\left(1-\frac{1}{m}\right)\left(u_{i j}(0)\right)+\left(\frac{1}{m}\right)\left(u_{i j}(1)\right)\right]=\sum_{j=1}^{n} \frac{1}{m} a_{i j}=\frac{1}{m} \sum_{j=1}^{n} a_{i j}=\frac{1}{m}
$$

where the last equality follows because all $i$ are assumed to have a utility of 1 for the whole cake.

Therefore,

$$
\sum_{j=1}^{n} u_{i j}\left(x_{i j}\right)<\frac{1}{m} \forall i \in M
$$

To prove that a proportional division may not even exist, consider $n=1$ with all $u_{i 1}(\cdot)$ still strictly convex. If all $x_{i 1}=\frac{1}{m}$, then no player receives a proportional allotment as per the previous logic. Else, $x_{i 1}<\frac{1}{m}$ for some $i=i^{\prime}$. By strict convexity, $u_{i^{\prime} 1}\left(x_{i^{\prime} 1}\right)<x_{i^{\prime} 1}$. Then by transitivity, $u_{i^{\prime} 1}\left(x_{i^{\prime} 1}\right)<\frac{1}{m}$.

This first theorem demonstrates that the proportionality criterion, which is often taken for granted in the cakecutting literature (see e.g., Brams et al., 2012), is not always achievable. Therefore, special attention needs to be given to this criterion when we allow utility functions to be nonadditive. The next theorem addresses this issue. 
Considering that there does not always exist a proportional allocation, an interesting question is whether there are any conditions that, if met by utility functions, guarantee the existence of a proportional allocation. In the following theorem, I provide sufficient conditions for the existence of a proportional allocation.

Theorem 2 If $m \geq n$ and, for all $i \in M$ and $j \in N$,

$$
\begin{aligned}
& \text { 1) } u_{i j}^{\prime}(p)>0, p \in \mathbb{R} \mid 0<p<1 \text {, and } \\
& \text { 2) } u_{i j}\left(\left\lceil\frac{m}{n}\right\rceil^{-1}\right) \geq \frac{1}{m} \text {, }
\end{aligned}
$$

then there exists a proportional allocation for the instance.

Proof. Sort the players into $n$ groups such that $(n-m \bmod n)$ of the groups consist of $\left\lfloor\frac{m}{n}\right\rfloor$ players each and $(m \bmod n)$ of the groups consist of $\left\lceil\frac{m}{n}\right\rceil$ players each.

Assign to each group $\mathcal{G}_{j}$ a unique component $j$. Thus, there is a set of $n$ groups $\left\{\mathcal{G}_{1}, \cdots, \mathcal{G}_{n}\right\}$.

Let $\# \mathcal{G}_{j} \equiv$ cardinality of $\mathcal{G}_{j}$. Set

$$
x_{i j}=\left\{\begin{array}{ll}
\frac{1}{\# \mathcal{G}_{j}}, & i \in \mathcal{G}_{j} \\
0, & i \notin \mathcal{G}_{j}
\end{array}, \forall j \in N .\right.
$$

By Condition 2 of the theorem, for $j \mid \# \mathcal{G}_{j}=\left\lceil\frac{m}{n}\right\rceil$, all players $i \in \mathcal{G}_{j}$ receive a proportional share. Next, for $j \mid \# \mathcal{G}_{j}=\left\lfloor\frac{m}{n}\right\rfloor$, each player $i \in \mathcal{G}_{j}$ derives from her allotment a utility of

$$
u_{i j}\left(\left\lceil\frac{m}{n}\right\rceil^{-1}\right)+\int_{\left\lceil\frac{m}{n}\right\rceil^{-1}}^{\left\lfloor\frac{m}{n}\right\rfloor^{-1}} u_{i j}^{\prime}(p) d p \geq \frac{1}{m}
$$

which completes the proof.

The next theorem relates to the concept of price (see Section 1.2). Caragiannis et al. (2012) place finite bounds on the prices of proportionality, envy-freeness, and equitability. However, they assume utility to be additive.

Theorem 3 The prices of envy-freeness and equitability are both unbounded with nonadditive $u_{i j}$ functions.

Proof. Consider an instance with $m=2$ and $n=1$. Define

$$
u_{11}(p)=u_{21}(p)=\frac{1+\alpha^{\beta}}{1+\left(\frac{p}{\alpha}\right)^{-\beta}}
$$

where $\alpha$ is the "median" and $\beta$ is the "shape" parameter (Note 13). Figure 4 shows such a utility function for $\alpha=.55$ and $\beta=20$.

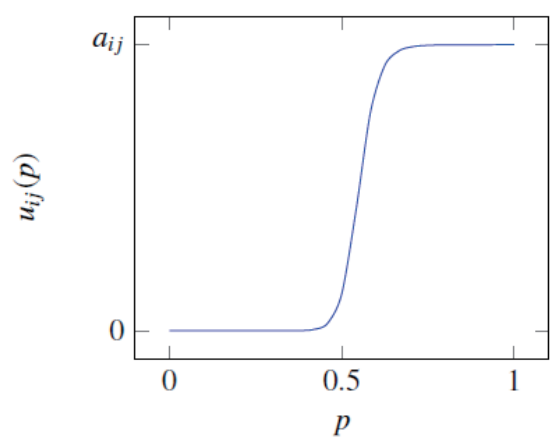

Figure 4. $u_{11}(p)=u_{21}(p)$ for $\alpha=.55$ and $\beta=20$

Now note that allocation $\mathbf{X}$ is guaranteed to be envy-free and equitable when $x_{11}=x_{21}=0.5$. Furthermore, it is easy to check that

$$
\lim _{\beta \rightarrow \infty} \frac{1+\alpha^{\beta}}{1+\left(\frac{0.5}{\alpha}\right)^{-\beta}}=0
$$


for any $\alpha>0.5$.

The above statements imply that

$$
\frac{u_{11}(1)}{2 \cdot \lim _{\beta \rightarrow \infty} \frac{1+\alpha^{\beta}}{1+\left(\frac{0.5}{\alpha}\right)^{-\beta}}} \rightarrow \infty
$$

where the numerator is the unconstrained societal utility and the denominator is the societal utility that satisfies fairness.

In contrast, assuming all utility functions to be additive, Caragiannis et al. (2012) prove the prices of proportionality and equitability to be $8-4 \sqrt{3}$ and $9 / 8$ respectively for $m=2$.

\section{Developing a Division Protocol That Accounts for Nonadditive Utility Functions}

\subsection{Types of Protocols}
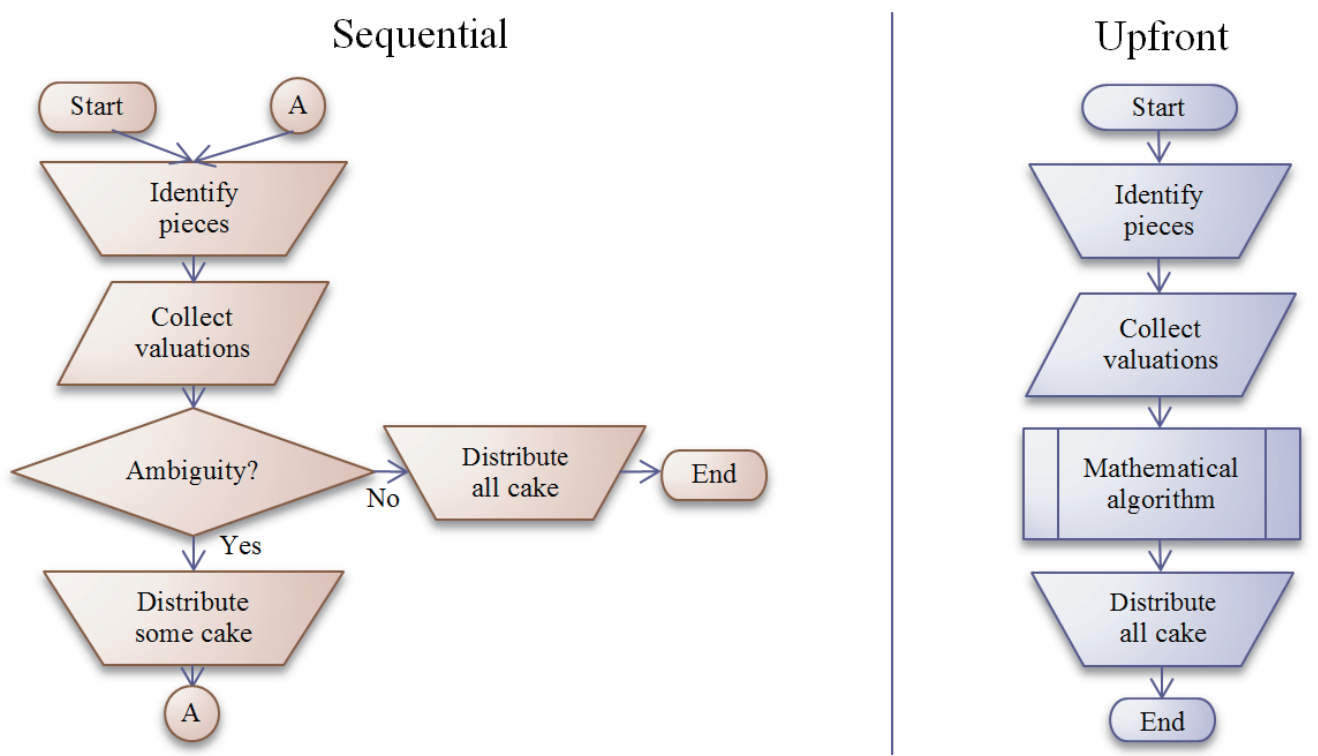

Figure 5. Analysis of the general classes of division protocols

I categorize the approaches for division protocols into two general classes, as shown in Figure 5. Not all protocols use numerical utility functions as I do in this work. In fact, most protocols are sequential: valuations are determined by using (essentially) binary input-in other words, a series of "yes" or "no" questions are posed to the players after all the pieces have been demarcated (Note 14) to determine preferences. The questions usually require the player to compare a certain piece with a numerical valuation and/or another piece. Sometimes these methods are also iterative: for example, in Kuhn's Method (see e.g. Borgers, 2010), which is designed to yield a proportional division, there may be a conflicted set of $\Upsilon$ players for whom there are only $v<\Upsilon$ pieces which at least one of the players considers proportional share. If this situation arises, the cake is distributed to those outside the conflicted set. Then, the remaining pieces are recombined. New pieces are demarcated and the process is repeated. With each cycle, the conflicted set becomes smaller. Eventually, the conflicted set has only two people, at which point the "I cut, you choose" procedure is used (recall in this method that one player divides the remaining cake into two pieces; the other chooses whichever piece she feels to be of more value).

My second classification for division protocols is "upfront". Such methods do not necessarily define numerical utility functions over the entire interval $[0,1]$, but they do collect numerical data. The numerical data is manipulated using a mathematical algorithm, yielding the fractions of each piece that are to be allocated to each player. A simple example of an upfront protocol is proportional allocation, designed to achieve equitability. Suppose players $A$ and $B$ are sharing a blueberry and strawberry tart. Their valuations for each component (in its entirety) are as shown in Table 1. 
Table 1. Valuation of two components by two players

\begin{tabular}{ccc}
\hline & Blueberry & Strawberry \\
\hline$A$ & 0.4 & 0.6 \\
$B$ & 0.2 & 0.8 \\
\hline
\end{tabular}

Since $A$ values the blueberry portion twice as much as $B$ does, $A$ is allocated twice as much of the blueberry portion. Specifically, $A$ receives $2 / 3$ of the blueberry portion and $B$ receives $1 / 3$. By the same logic, $A$ receives $3 / 7$ of the strawberry portion and $B$ receives $4 / 7$. If utility is additive, the allocation is clearly equitable (Note 15) since

$$
\frac{2}{3} \times 0.4+\frac{3}{7} \times 0.6=\frac{1}{3} \times 0.2+\frac{4}{7} \times 0.8 \approx 0.524
$$

But if we allow utility to be superadditive or subadditive, then the above equation does not accurately represent total utility for either player, and so this becomes another protocol that fails (in general) to meet its objective.

In constructing my own method to handle superadditive and subadditive utility, I chose to use an "upfront" approach for two reasons:

1) Practicality: In real-world conflict resolution, it can be difficult to coordinate multiple valuation collections. For more bitter conflicts, the players may not wish to interact with each other; but using a sequential method, they would have to examine the "cake" multiple times. As a result, the process would become more time-consuming, more difficult to coordinate, and more unseemly for practical application.

2) Flexibility: Using a mathematical algorithm allows numerical utility functions to be given as input. These functions need not be linear-they can take any form, provided that the function is defined on $[0,1]$ and the other conditions discussed in Section 2.3 are met. In such a manner, the utility functions will be the most accurate models of utility possible.

\subsection{Using Mathematical Programming to Find a Fair Division}

Table 2. An example of an instance

$$
\begin{array}{ll}
\hline u_{11}(p) & 0.3\left[3(p)-2(p)^{2}\right] \\
u_{12}(p) & 0.4\left[2(p)^{3}-p\right] \\
u_{13}(p) & 0.3\left[\left(1-e^{-p}\right) /\left(1-\frac{1}{e}\right)\right. \\
u_{21}(p) & 0.3[\sqrt{p}] \\
u_{22}(p) & 0.5\left[-3(p)+4(p)^{2}\right] \\
u_{23}(p) & 0.2\left[(p)^{2}\right] \\
u_{31}(p) & 0.4\left[(p)^{4}\right] \\
u_{32}(p) & 0.3\left[-(p-1)^{4}+1\right] \\
u_{33}(p) & 0.3\left[2(p)-(p)^{2}\right] \\
\hline
\end{array}
$$

*Note that the $a_{i j}$ have been factored out, so the expressions in brackets have a value of 1 for $p=1$.

The "upfront" method that I elected to use was nonlinear programming (Note 16). This approach allowed me to enter the $x_{i j}$ as the decision variables and base my objective and constraints on the $u_{i j}$ functions. Table 2 shows one of the instances that I experimented with. It has $m=3$ and $n=3$.

Notice the variety of functions that may be used. Included in this instance are quadratic, cubic, quartic, square root, and exponential functions.

Identifying Constraints. In order to bound the feasible region, some constraints must be established. There are two sets of constraints on the $x_{i j}$ values:

1) $x_{i j} \geq 0 \forall i \in M, \forall j \in N$, and

2) $\sum_{i \in M} x_{i j}=1, \forall j \in N$. 
The fairness criteria of proportionality, envy-freeness, and equitability can also be guaranteed by the following constraints:

- Proportionality: $\sum_{j=1}^{n} u_{i j}\left(x_{i j}\right) \geq \frac{1}{m}, \forall i \in M$

- Envy-freeness: $\sum_{j=1}^{n} u_{i j}\left(x_{i j}\right) \geq \sum_{j=1}^{n} u_{i j}\left(x_{k j}\right), \forall i, k \in M$

- Equitability: $\sum_{j=1}^{n} u_{i j}\left(x_{i j}\right)=\sum_{j=1}^{n} u_{k j}\left(x_{k j}\right), \forall i, k \in M$

It is easy to show that, in general, these three criteria cannot be satisfied concurrently. For some instances, it may not be possible to meet any at all. It may also be possible that the criteria can be met, just not concurrently (Note 17). Depending on the instance, the players may have to pick and choose between criteria. This may seem to be a pessimistic result, but one may also take the stance that it is better to prove the impossibility of meeting a set of criteria than to give an inaccurate solution based on the assumption of additive utility.

Identifying the Objective Function. For some instances, there will be multiple solutions that all satisfy the desired criteria. Therefore, the objective function can be set to maximize total societal utility:

$$
\max _{\mathbf{X}} \sum_{i=1}^{m} \sum_{j=1}^{n} u_{i j}\left(x_{i j}\right) .
$$

For certain situations, however, another objective function may be more appropriate. Recall the notion of Paretooptimality from Section 1.2. This is another key desideratum, but it does not have its own mathematical programming constraint. However, Willson (1995) shows that with additive utilities, Pareto-optimality can be achieved through the following objective (see also Borgers, 2010):

$$
\max _{\mathbf{X}}\left\{\min _{i=1,2, \ldots, m} \sum_{j=1}^{n} u_{i j}\left(x_{i j}\right)\right\} .
$$

This result does not directly extend to instances with nonadditive utility functions. The following theorem identifies conditions under which this result extends to nonadditive utility functions.

Theorem 4 Assume that $u_{i j}(p)>0 \wedge u_{i j}^{\prime}(p)>0, x_{i j} \in \mathbb{R} \mid 0<x_{i j} \leq 1, \forall i, j$. A division is Pareto-optimal and equitable if and only if it maximizes the minimal share, regardless of whether utility is additive.

Proof.

1) If a division is Pareto-optimal and equitable, then it maximizes the minimal share.

Assume not. Then there must be an alternative division with greater minimal share. Since the given division is equitable, the alternative division would be an objective improvement, which contradicts Pareto-optimality.

2) If a division maximizes the minimal share, then it is Pareto-optimal and equitable.

Assume the division is not equitable. Then those with maximal share could distribute a small amount of their cake to others, which would necessarily increase the minimal share (due to the assumption regarding the derivative of $u_{i j}$ ). This contradicts our original assumption that the division maximizes the minimal share. Assume an objective improvement can be made. Since the division, as we have already proved, is equitable, the objective improvement would improve everyone's utility. This contradicts our original assumption that the division maximizes the minimal share.

Example Allocation. Figure 6 shows a spreadsheet with the sample problem and the optimal envy-free and equitable allocation. Figure 7 shows the constraints for (a) bounds on $x_{i j}$, (b) envy-freeness, and (c) equitability as programmed into Risk Solver Platform Version 12.

The proposed allotment is given in cells L7:N9 (the decision variables). For example, about 13\% of component 1 goes to player 1. Notice that in cells L16, M17, and N18, the value of .3772 is displayed. This means that each player feels she receives a utility of .3772 from her own allotment. This is a highly satisfactory division: not only were envy-freeness and equitability met; so was proportionality-in fact each player's utility for her own allotment exceeds $1 / 3$. 


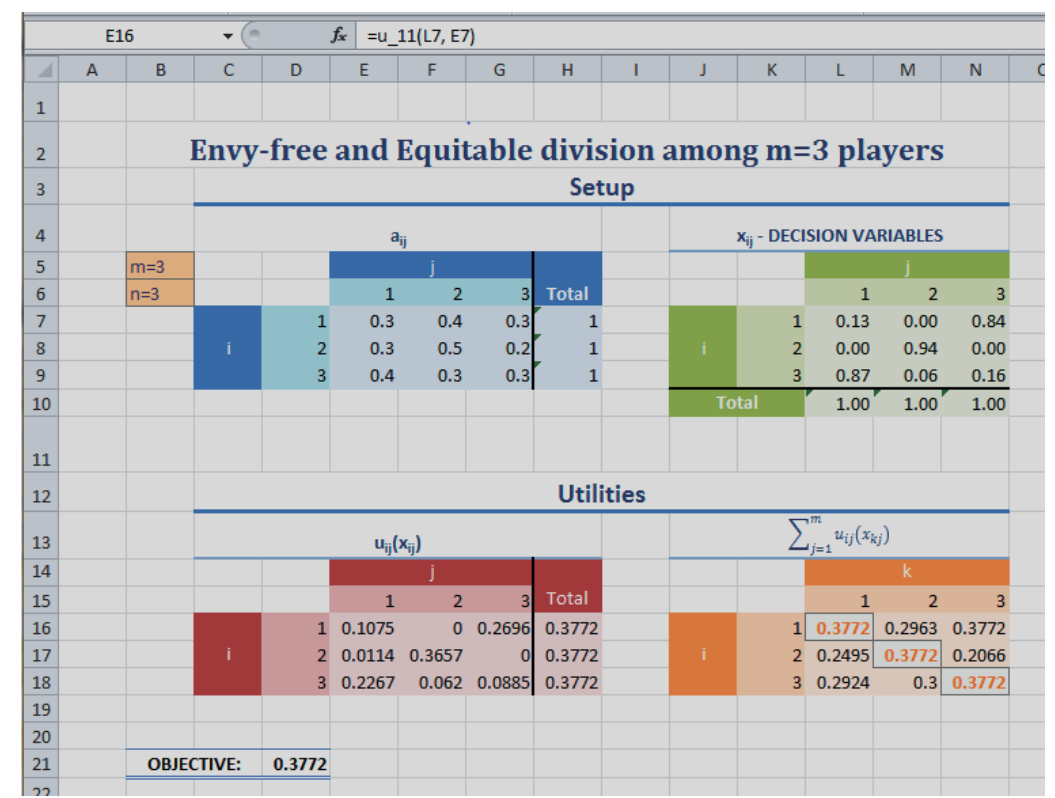

Figure 6. Spreadsheet showing the utility functions, the proposed allotment, and the utilities resulting from the proposed allotment

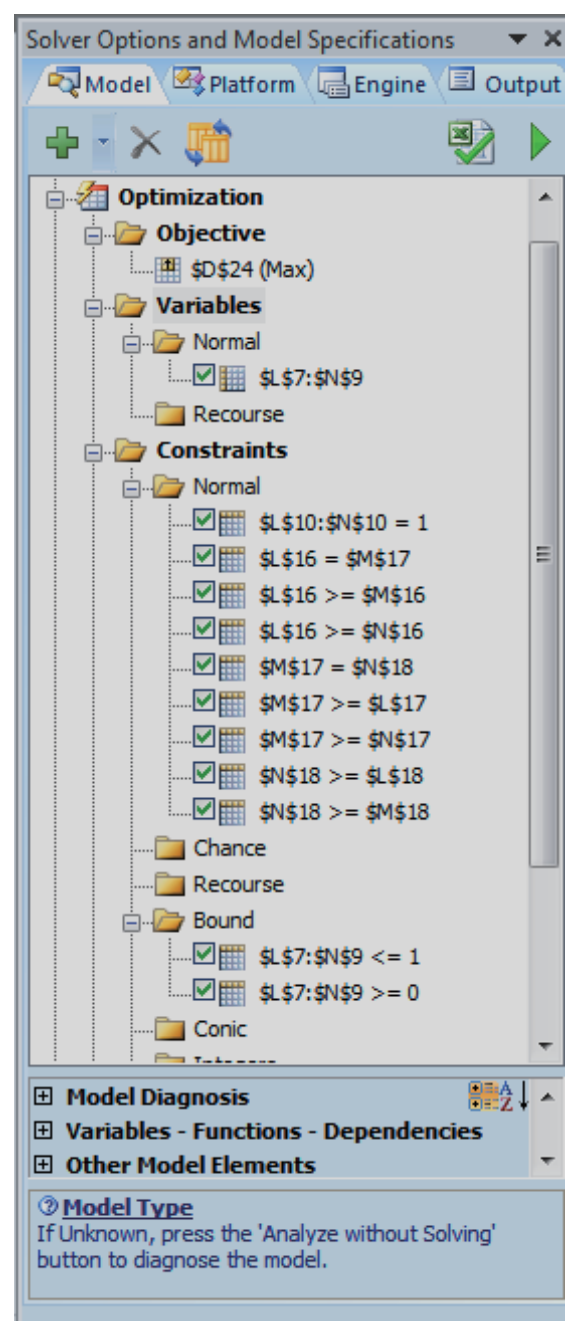

Figure 7. Panel from Risk Solver Platform showing objective, decision variables, constraints, and bounds 


\subsection{Interacting Components}

My approach easily incorporates situations where two (or more) components interact to affect the value of their union. To see this, define the total utility of allocation $\mathbf{X}$ to player $i$ as follows:

$$
U_{i}(\mathbf{X})=\sum_{j=1}^{n} u_{i j}\left(x_{i j}\right)+\sum_{j=1}^{n-1} \sum_{k=j+1}^{n}\left[\theta_{i, j k}\left(x_{i j}, x_{i k}\right)\right] .
$$

The first addend on the right hand side of the equal sign is the total utility from the individual components-without the interaction effect considered. (Note that as previously discussed, each of the $u_{i j}$ functions may be nonadditive.) The second addend is where the interaction effect may come in.

Let $p$ and $q$ represent amounts of components $j$ and $k$, respectively. If, for player $i, \theta_{i, j k}(p, q) \neq 0$ for some $0<p, q \leq 1$, then there is an interaction effect for player $i$ with respect to components $j$ and $k$.

As an example, consider three players $A, B$, and $C$ trying to divide a supply of milk and cookies. $A$ is allergic to milk and thus only derives utility from cookies. $B$ can derive utility from both milk and cookies, but much prefers to have some of both so that she can dunk the cookies. $C$ can also derive utility from both milk and cookies, but doesn't really like to dunk the cookies. In such a situation, the utility functions may look as in Table 3 .

Table 3. An Instance with complementary components

\begin{tabular}{llll}
\hline & Milk $(j=1)$ & Cookies $(j=2)$ & Interaction Effect \\
\hline Player $A(i=1)$ & $u_{11}(p)=0$ & $u_{12}(q)=1-(1-q)^{4}$ & $\theta_{1,12}(p, q)=0$ \\
Player $B(i=2)$ & $u_{21}(p)=\frac{1}{4} p$ & $u_{22}(q)=\frac{1}{4} q$ & $\theta_{2,12}(p, q)=\frac{1}{2} p q$ \\
Player $C(i=3)$ & $u_{31}(p)=\frac{1}{2} q$ & $u_{32}(q)=\frac{1}{2} q$ & $\theta_{3,12}(p, q)=0$ \\
\hline
\end{tabular}

In this example, the milk and cookies are complementary for Player B. But the interaction effect could work the opposite way when two items are mutually incompatible. Consider two patients $D$ and $E$. Both patients are suffering from allergies and would derive utility from antihistamines. $E$ also has difficulty sleeping and so would derive utility from some sedative. However, $E$ cannot take both antihistamines and sedatives simultaneously, for this combination would dangerously affect her concentration level. Thus, the utility functions may look as in Table 4.

Table 4. An Instance with mutually incompatible components

\begin{tabular}{llll}
\hline & Antihistamines $(j=1)$ & Sedatives $(j=2)$ & Interaction Effect \\
\hline Player $D(i=1)$ & $u_{11}(p)=3 p-2 p^{2}$ & $u_{12}(q)=0$ & $\theta_{1,12}(p, q)=0$ \\
Player $E(i=2)$ & $u_{21}(p)=3\left(3 p-2 p^{2}\right)$ & $u_{22}(q)=3\left(3 q-2 q^{2}\right)$ & $\theta_{2,12}(p, q)=-5 p q$ \\
\hline
\end{tabular}

Both of these examples demonstrate the second-order interaction effect, but the concept is easily generalized to higher orders for instances with $n>2$.

None of the existing fair division models capture such an interaction effect. Since there are many plausible instances where two components affect each other's value, this ability of my algorithm is useful.

\section{Conclusions and Directions for Future Research}

\subsection{Summary of Contributions}

The objective of this investigation was to examine the implications of a problematic assumption, that of additivity in utility, in the fair division problem. In this work, I accomplished the following:

- Identified a problematic assumption in the fair division literature.

- Proposed a new way of formalizing the problem. 
- Proposed a mathematical-programming-based division protocol for nonadditive utility functions.

- Investigated the relationship between envy-freeness and proportionality (Theorem 1).

- Established conditions guaranteeing the existence of a proportional allocation (Theorem 2).

- Proved that the price of fairness can be unbounded (Theorem 3).

- Demonstrated how to achieve Pareto-optimality and equitability (Theorem 4).

- Explored the possibility of components interacting to affect the value of their union.

\subsection{Open Questions}

Necessary conditions for the existence of a proportional allocation. Theorem 3 presents sufficient conditions for the existence of a proportional allocation. However, the result does not tell when a proportional allocation does not exist. Since proportionality is an important fairness criterion, it would useful to establish necessary conditions for the existence of a proportional allocation.

Specialized algorithms. My division protocol can easily incorporate many different types of utility functions, including those with the interaction effect. However, I recognize that nonlinear programming can be computationally challenging in general. Accordingly, specialized algorithms may need to be developed for specific types of nonadditive utility functions.

A Pareto-optimal and envy-free protocol that allows for nonadditive utility functions. Even with additive utility functions, meeting Pareto-optimality and envy-freeness concurrently is extremely difficult. Reijnierse and Potter (1998) create a market environment to achieve these two desiderata for additive utility functions: players start out with the same amount of money and bid on the various homogeneous components. It remains to be proven whether such a concept can be extended to nonadditive utility functions.

A more robust way to achieve Pareto-optimality. In Theorem 1, it was assumed that $u_{i j}(p)>0 \wedge u_{i j}^{\prime}(p)>0, p \in$ $\mathbb{R} \mid 0<p \leq 1, \forall i, j$. These assumptions limit the number of instances for which the objective of maximizing the minimal share is enough to ensure Pareto-optimality. It would be useful to have a more broadly applicable way to achieve Pareto-optimality.

\section{Acknowledgements}

I would like to express my sincere appreciation for the committed guidance of my mentor, Richard E. Wendell, Ph. D. at the University of Pittsburgh. I would also like to thank Prakash B. Mirchandani, Ph. D. at the University of Pittsburgh and Mihai M. Banciu, Ph. D. of Bucknell University for their comments on this work. Finally, I would like to thank Society for Science and the Public and all my judges and fellow competitors at the 2013 Intel International Science and Engineering Fair (ISEF) for giving me an incredibly diverse and intellectually stimulating forum at which to present this research.

\section{References}

Aumann, R., \& Maschler, M (1985). Game theoretic analysis of a bankruptcy problem from the Talmud. Journal of Economic Theory, 36(2), 195-213. http://dx.doi.org/10.1016/0022-0531(85)90102-4

Aumann, Y., \& Dombb, Y. (2011). The efficiency of fair division with connected pieces. In A. Saberi (Ed.), Internet and Network Economics. Paper presented at WINE '10: 6th International Workshop, Stanford University (pp. 26-37). Germany: Springer Verlag Heidelberg. http://dx.doi.org/10.1007/978-3-642-17572-5_3

Brams, S. J., \& Taylor, A. (1995). An envy-free cake division protocol. The American Mathematical Monthly, 102(1), 9-18. http://dx.doi.org/10.2307/2974850

Börgers, C. (2010). Mathematics of social choice: Voting, compensation, and division. Philadelphia: Society for Industrial and Applied Mathematics.

Brams, S. J. (2008). Mathematics and democracy: Designing better voting and fair division procedures. Princeton, N.J.: Princeton University Press.

Brams, S. J., Feldman, M., Lai, J., Morgenstern, J., \& Procaccia., A. (2012). On Maxsum Fair Cake Divisions. Paper presented at Twenty-Sixth AAAI Conference on Artificial Intelligence, Toronto (pp. 1285-1291). AAAI Press.

Caragiannis, I., Kaklamanis, C., Kanellopoulos, P., \& Kyropoulou, M. (2012). The Efficiency of Fair Division. 
Theory of Computing Systems, 50(4), 589-610. http://dx.doi.org/10.1007/s00224-011-9359-y

Gerber, H., \& Parfumi, G. (1998). Utility functions: From risk theory to finance. North American Actuarial Journal, 2, 74-91. http://dx.doi.org/10.1080/10920277.1998.10595728

Johnson, N. L., Kotz, S., \& Balakrishnan, N. (1994). Continuous univariate distributions, Vol. 2. New York: Wiley.

Koutsoupias, E., \& Papadimitriou, C. (1999). Worst-case equilibria. In C. Meinel, \& S. Tison (Eds.), Lecture Notes on Computer Science, Vol. 1563. Paper presented at STACS '99, Trier, Germany (pp. 404-413). Germany: Springer.

Moulin, H. (2003). Fair division and collective welfare. Cambridge: MIT Press.

Petersen, E., \& Su, F. E. (2002). Four-person envy-free chore division. Mathematics Magazine, 75(2), 117-122.

Ragsdale, C. T. (2012). Spreadsheet modeling \& decision analysis: A practical introduction to management science. Mason, OH: South-Western, Cengage Learning.

Reijnierse, J. H., \& Potters, J. A. M. (1998). On finding an envy-free Pareto-optimal division. Mathematical Programming, 83(1), 291-311. http://dx.doi.org/10.1007/BF02680564

Steinhaus, H. (1948). The problem of fair division. Econometrica, 16(1), 101-104.

Traxler, M. (2002). Fair chore division for climate change. Social Theory and Practice, 28(1), 101-134. http://dx.doi.org/10.5840/soctheorpract20022814

Willson, S. J. (1995). Fair division using linear programming. Unpublished manuscript, Iowa State University.

Winston, W. L. (1991). Introduction to mathematical programming: Applications and algorithms. Boston: PWSKENT.

\section{Notes}

Note 1. The Talmud (c. 200-500 C.E.) discusses fair division in several contexts. See, e.g., Aumann and Maschler, 1985.

Note 2. Previous work (e.g., Reijnierse \& Potters, 1998; Brams et al., 2012) considered utility functions as nonatomic value density functions defined on the entire area of the cake (i.e., for a given player, the utilities for all components were represented by a single accumulation function). These value density functions were classified as piecewise constant.

I do not use the concept of value density in this work. Since, in my formalization of the problem, separate utility functions are defined for every possible combination of individual $i$ and component $j$, defining the $u_{i j}$ as accumulation functions would be unnecessary. (It would also make the division protocol I propose in Section 4.2 significantly more difficult to solve.) The condition of piecewise constancy manifests itself as additivity in my system.

Note 3. Quantity of allotment can imply length, area, volume, or weight (among other measures) depending on the object being divided. For example, allotment of a piece of land would be measured by area, allotment of a medicine would be measured by weight, etc.

Note 4. The distinction between envy-freeness and equitability is as follows: Equitability requires that each player responds with the same answer to the question "What fraction of the total value of the cake do you think you are receiving?" On the other hand, envy-freeness allows, say, player 1 to give a higher answer to this question than player 2-as long as player 2 disagrees with player 1's judgment; i.e., player 2 values player 1's allotment at most as much as her own allotment.

Note 5. I note that this concept of the price of fairness is related to the concept of the price of anarchy introduced by Koutsoupias and Papadimitriou (1999).

Note 6. Note that it is not necessary to define the price of Pareto-optimality since it is equal to one for all instances. This is because the most efficient Pareto-optimal allocation is always the optimal allocation for the instance.

Note 7. In the Hindu epic Ramayana (c. 4th century BCE), the sage Valmiki describes the banishment by King Dasaratha of Lord Rama, his eldest son and rightful heir, for 14 years so that another of his sons could become the king in the interim. This is also an example of division, though temporal rather than the commonly studied spatial. 
Note 8. Actuarial scientists have considered monetary utility functions (see, e.g, Gerber \& Parfumi, 1998). These functions are assumed to be nonnegative, monotonically increasing, and if risk-averseness holds, concave. We shall see that these conditions do not necessarily hold for goods.

Note 9. As opposed to half the utility of the full course of antibiotics, which would have been the result given by an additive utility function.

Note 10. http://www.mayoclinic.com/health/antibiotics/FL00075/NSECTIONGROUP=2. Accessed 17 June 2013.

Note 11. I assume $u_{i j}$ to be differentiable for the purpose of explaining this concept; I will soon discuss nondifferentiable utility functions.

In examining situations where utility is nonadditive, I not only allow that the $u_{i j}$ may be nonlinear but also that they may be discontinuous. (This is why I use the more general terms "additivity", "subadditivity", and "subadditivity" instead of "linearity", "convexity", and "concavity", respectively.)

Note 12. I assume that not all utility functions behave in this discontinuous manner. Otherwise, the cake would become a single indivisible object that could only be allocated to one person (or else no one at all would receive any utility). In this situation, a compensation algorithm would be more appropriate (see e.g. Borgers, 2010).

Note 13. To obtain this utility function, I start with the log-logistic function (Johnson et al., 1994) and normalize it so that it equals one when $p=1$. I use this function for its manipulability: By changing the median and shape parameters, we can model a wide range of utility characterizations with this function.

Note 14. This demarcation may seem to be a trivial step. However, in some sequential methods, the demarcations of the components are not the same as the demarcations of the pieces for which valuations are collected. For example, Kuhn's method begins with a lone divider dividing the cake into $m$ pieces, each of which may incorporate pieces of multiple components. So if a cake has a strawberry component, a vanilla component, and a chocolate component, a possible piece could be $3 / 4$ of the strawberry, $1 / 8$ of the vanilla, and $1 / 7$ of the chocolate.

Note 15. See Borgers (2010) for a formal proof that, in general, proportional allocation for $m=2$ players is necessarily equitable with additive utility.

Note 16. I base my approach off of Willson (1995) who uses linear programming to find fair divisions. However, he defines the utility functions for all $i$ and $j$ as $u_{i j}(p)=a_{i j} \cdot p$, thus requiring additive utility. Since I change the $u_{i j}$ to make them nonlinear, I cannot use linear programming methods such as the simplex method. Instead, I use nonlinear programming.

Note 17. With additive utility, every instance has at least one allocation to meet any given fairness criterion. The equal division, in which an equal quantity of every component is allotted to each player, guarantees proportionality, envy-freeness, and equitability. This clearly is not necessarily the case if utility can be nonadditive.

\section{Copyrights}

Copyright for this article is retained by the author(s), with first publication rights granted to the journal.

This is an open-access article distributed under the terms and conditions of the Creative Commons Attribution license (http://creativecommons.org/licenses/by/3.0/). 